\title{
A Reversible Data Hiding Scheme Using Ordered Cluster-Based VQ Index Tables for Complex Images
}

\author{
Junlan Bai ${ }^{1}$, Chin-Chen Chang ${ }^{2}$, and Ce Zhu ${ }^{1(\sqrt{ })}$ \\ ${ }^{1}$ University of Electronic Science and Technology of China, Chengdu, China \\ 907162937@qq.com, eczhuluestc.edu.cn \\ ${ }^{2}$ Feng Chia University, Taichong, Taiwan \\ alan3c@gmail.com
}

\begin{abstract}
In the digital multimedia era, data hiding in the compression domain becomes increasingly popular in the need for speeding up the transmission process and reducing bandwidth. Recently, VQ-based watermarking techniques have attracted more attentions, e.g., Tu and Wang proposed a VQ-based lossless data hiding scheme with high payload most recently. However, their scheme produces some more overhead information. In this study, we develop a novel reversible hiding scheme which may reduce the use of overhead bits, which is especially effective for images of complex texture. Specifically, a codebook is partitioned into six clusters which are organized based on the usage frequency of codewords. We then develop a new mapping relationship among the six clusters to hide secret data more cost-effectively. The experimental results demonstrate that our proposed scheme outperforms Tu's scheme for complex texture images.
\end{abstract}

Keywords: Reversible data hiding · Vector Quantization (VQ) · Data clustering · Image compression

\section{Introduction}

In recent decades, with the booming growth of wireless network and worldwide telecommunication, people tend to become more and more reliant on Internet. However, it will result in some potential problems generated by digitalized data without the assurance of security and protection of confidentiality [1]. The information security has become an increasingly important issue to be protected against illegal three-party access. Many data hiding algorithms [2-4] have won popularity in terms of its imperceptibility, undetectability and invisibility. Data hiding is treated as embedding data or watermarking into a host media or cover media such as texts, images, videos and so on. In this paper, we mainly focus on steganography based on digital images.

The secret data can be embedded into three domains of the cover image, named the spatial domain, the frequency domain, and the compression domain, respectively. The image compression techniques have won great popularity. Among them, VQ [5-8] is considered as a most attractive compression method and embraces extensive popularity because of its simplicity and usability for grayscale images compared with other 
compression schemes. Thus, many scholars have devoted themselves to doing research on data hiding techniques based on VQ compression for diverse digit images [9-12]. Jo and Kim proposed a data hiding scheme based on VQ compression for digital images in 2002 [9]. It takes advantage of the similarity that the codebook trained by LBG algorithm [5] includes many similar codewords. This method is lack of reversibility and suffers from limited payload which restricts the quantity of information transmitted in the channel. Subsequently, a reversible data hiding scheme [10] which emphasizes recovering the VQ compressed codes index was proposed by Chang et al. in 2007. However, it has a considerably low hiding capacity and rather high cost since only one third of the VQ codewords can conceal secret data and it brings in extra bits as many as the size of codebook.

In 2014, Tu and Wang presented a lossless data hiding with high payload based on VQ compression [11] which further extends Chang et al.'s method [10]. For notation simplicity, we denote Tu and Wang's scheme in [11] by Tu's scheme. Their scheme tries to embed secret data for each group by partitioning the codebook into three clusters in advance according to its referred frequency of VQ compressed code index. The second highest cluster and the last one (i.e., cluster $_{2}$ and cluster $_{3}$ ) both require an extra bit to distinguish the VQ indices between the two clusters. In particular, when the complexity of the texture of image increases, the cost will also rapidly grow since more and more indices are distributed in cluster ${ }_{2}$ and cluster $_{3}$.

Inspired from Tu's method [11], a novel reversible data hiding scheme based on clustering according to the referred frequency of VQ code indices is developed in this paper. The proposed method can save the number of bits on cost more largely than Tu's scheme for complex images.

The rest of the paper is organized as follows. An elaborate description of our proposed scheme will be fully exposed in Sect. 2. In Sect. 3, experimental investigation of the rules and relationship in various kinds of images, as well as different codebook sizes are performed to show that our scheme outperforms Tu's for complex images. At last, we present our conclusions in Sect. 4.

\section{Proposed Algorithm}

For the sake of reducing the expenses and meanwhile keeping the payload as high as Tu's method, we propose a novel reversible data hiding scheme based on VQ. In our proposed method, we reduce the usage of extra bits by exploiting the newly constructed mapping relationship. The proposed algorithm phase is comprised of three parts: pre-processing phase, embedding phase, and extraction and decompression phase, respectively.

\subsection{The Pre-processing Phase}

This phase aims at obtaining the re-ordered VQ codebook of specified size to contribute to the future embedding procedure. The original codebook $C B$ is generated by the LBG training algorithm [5] in advance. Each test image is firstly segmented into a set of non-overlapping blocks where all of them have been encoded by VQ to determine the best-matched codeword in the well-trained codebook $C B$ by computing the minimum Euclidean distance. The index value of the most similar 
codeword is taken as the compressed index which replaces each block. Consequently, the quantizer will generate an index table where the size of the original image is significantly reduced. The detailed algorithm is presented below.

The referred frequencies counts of the $K$ codewords are initially set to zero. While the nearest codeword of an image block has been confirmed, the referred frequency of this codeword in the codebook is increased by 1 . Count for the total number of occurrence of each codeword and put the original codebook $C B$ in descending order in terms of the referred frequency of each codeword. We mark the new descending ordered codebook as $C B_{1}$. The front $\lfloor K / 6\rfloor \times 6$ codewords in the sorted codebook $C B_{1}$ are picked up to constitute the new codebook $C B_{2}$ where the rest $K-\lfloor K / 6\rfloor \times 6$ codewords are discarded. Herein, the new codebook $C B_{2}$ is divided into six clusters on average which consist of the same size of $m(m=\lfloor K / 6\rfloor)$ and are sequentially denoted as $\mathrm{C}_{\mathrm{i}}(i=1,2$, $\ldots, 6)$. The first cluster called $C_{1}$ is built by the $m$ highest referred frequencies, and then those with the next $m$ higher frequencies of referred codewords form the second cluster $\mathrm{C}_{2}$, and so on, until all of the six clusters have been successively set up, where $\mathrm{C}_{6}$ consists of the lowest referred frequencies of the codewords.

\subsection{The Embedding Phase}

In the embedding phase, we are going to process the VQ index table (i.e., VQ-image). Consider that each index hides one secret bit. The embedding manipulation steps are described below.

At first, we need figure out which cluster the input index is situated in. Once the corresponding cluster is determined, start to embed secret data obedient to the following rules.

(a) The input index belongs to $\mathrm{C}_{1}$.

(i) If the input index is going to carry secret bit 0 , the index should straightly convert into the corresponding index in $\mathrm{C}_{2}$.

(ii) If the input index is going to carry secret bit 1 , the index should straightly convert into the corresponding index in $\mathrm{C}_{3}$.

(b) The input index belongs to $\mathrm{C}_{2}$.

(i) If the input index is going to carry secret bit 0 , the corresponding index in $\mathrm{C}_{1}$ should replace the current input index.

(ii) If the input index is going to carry secret bit 1 , the corresponding index in $\mathrm{C}_{4}$ should replace the current input index and an extra bit ' 1 ' needs to be attached to the rear of the corresponding index. The indicator ' 1 ' means the current index is transformed from other clusters.

(c) The input index belongs to $\mathrm{C}_{3}$.

(i) If the input index is going to carry secret bit 0 , the corresponding index in $\mathrm{C}_{5}$ should substitute for the current input index.

(ii) If the input index is going to carry secret bit 1 , the corresponding index in $\mathrm{C}_{6}$ should substitute for the current input index.

Both the two cases in (c) need an extra bit ' 1 ' to be attached to the rear of the corresponding index which also means the current index is formed by transformation. 
(d) The input index is situated in $\mathrm{C}_{4}, \mathrm{C}_{5}$ or $\mathrm{C}_{6}$.

In such cases, there is not transformation any more. Whether the secret data is 0 or 1 , the index remains unchanged. An extra bit ' 0 ' needs to be attached to the index to indicate that the current index comes from itself rather than transformation from the other clusters. Additionally, the secret data also needs to be directly attached to the rear of the indicator. To explain it more clearly, the current index $I$ is encoded as the form $I \| \underline{0} s$, where $\|$ is the concatenation operation notation, the underlined ' 0 ' next to the concatenation notation is considered as the indicator bit and $s$ represents the one-bit secret data, respectively.

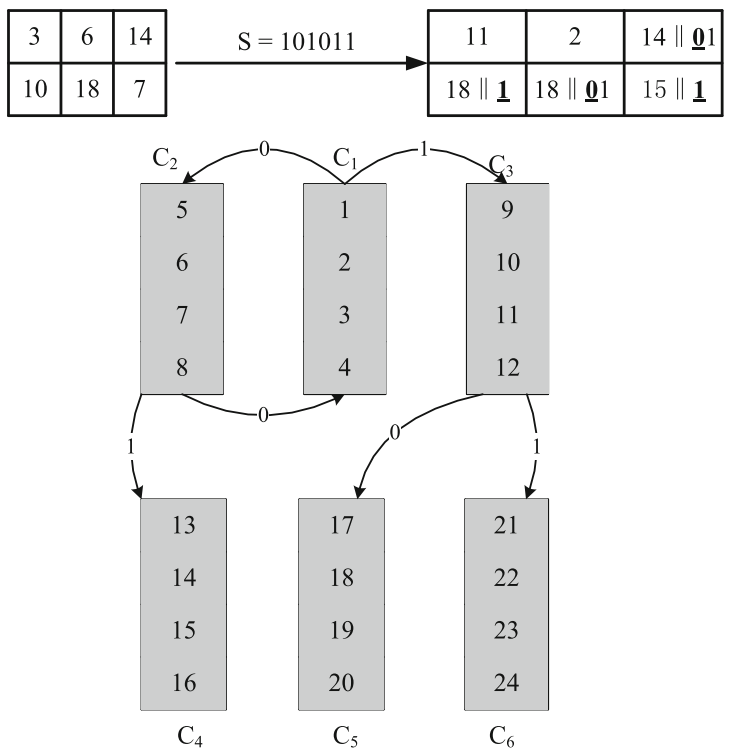

Fig. 1. Example of hiding one-bit secret data by the proposed scheme

Figure 1 depicts an example of the proposed embedding scheme. In this example, one-bit secret is hidden into each index. Assume that the original codebook size is 28 and the re-organized codebook contains 24 codewords instead which are then segmented into six clusters of the same size 4 in descending order as shown in Fig. 1.

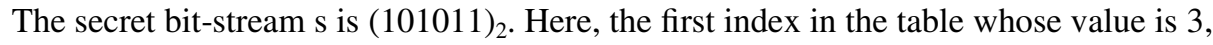
to be located in $\mathrm{C}_{1}$, carries the one-bit secret data 1 . Then the index value 3 is converted into the value 11 in $\mathrm{C}_{3}$ based on the mapping relationships. Likewise, the second index value 6 in $C_{2}$ which is going to embed secret data ' 0 ' is switched into the value 2 in $\mathrm{C}_{1}$ according to the embedding rules. And next, the third index value 14 situated in $\mathrm{C}_{4}$ which carries the two-bit secret data ' 1 ' remains unchanged since it belongs to $\mathrm{C}_{4}$. According to the designed embedding rules, we attach an extra bit ' 0 ' to the rear of the index 14, and the secret data ' 1 ' is additionally attached to the rear of indicator of the corresponding index. Similarly, the rest of the indices in table to embed secrets are almost the same as the above operation. The final resulting index table after embedding is shown in Fig. 1. 


\subsection{The Extraction and Decompression Phase}

The extraction and decompression process is just the inverse process of the embedding phase. In this phase, the receiver should obtain the codebook $\mathrm{CB}_{2}$ either in public or in private at first. Subsequently, the codebook is equally segmented into six clusters in the same way as embedding phase. The secret data extraction procedure merely requires simple cluster look-up operation. Based on this concept, we exploit the existence of the indicator to perform the decoding process below in detail.

(a) If the index is situated in $\mathrm{C}_{1}$, the extracted secret data is 0 and the index is recovered by the corresponding index in $\mathrm{C}_{2}$.

(b) If the index belongs to $\mathrm{C}_{2}$ or $\mathrm{C}_{3}$, then it is directly transformed into the corresponding index value in $\mathrm{C}_{1}$, and the extracted secret data is 0 and 1 , respectively.

(c) If the index is located in $\mathrm{C}_{4}, \mathrm{C}_{5}$ or $\mathrm{C}_{6}$, two cases must be considered. Firstly we need to check out whether the one bit that follows the index is ' 0 ' or ' 1 '.

(i) If the indicator bit is ' 1 ', we can ascertain the current index is from other clusters. If the index is situated in $\mathrm{C}_{4}$, the corresponding index in $\mathrm{C}_{2}$ will replace it and the extracted secret data is ' 1 '. Except for this situation, when the index belongs to $\mathrm{C}_{5}$ or $\mathrm{C}_{6}$, it is directly transformed into the corresponding index value in $\mathrm{C}_{3}$, and the hidden secret data is 0 and 1 , respectively.

(ii) If the indicator bit is ' 0 ', the index doesn't need modification any more for reversion since the current index is from itself and the secret bit is achieved by extracting the bit followed by the indicator of the index.

\section{Experimental Results}

In this section, performances of the proposed scheme are presented from both historical and vertical analyses and the comparisons with the Tu's VQ-based method are described to demonstrate the superiority of the proposed scheme for complex images. All the experiments are conducted by nine grayscale images: smooth images (e.g., 'Jet', 'Peppers', 'Tiffany'), high texture images (e.g., 'Barbara', Boat', 'Lake', 'People'), and extremely high texture images (e.g., 'Baboon' and 'Bridge'), where all of them are of the same size $512 \times 512$ as shown in Fig. 2 . The codebook that contains $256,512,1024$ codewords of 16-dimension are trained by the classical LBG algorithm, in which 'Lena', 'Baboon', 'Jet', 'Barbara' are employed as the training images. All of these images are encoded by VQ compression and will generate 16384 indices since the image size is equal to $512 \times 512$ and each of them is partitioned into $4 \times 4$ blocks. Denote that one bit secret is embedded into each index and the secret data is a binary bit steam which is created by a pseudo-random number generator.

We mainly concentrate on analyzing the contrast performance between our proposed scheme and Tu's method [11] in terms of the total hiding capacity, the cost and the actual neat hiding capacity, since Tu's method has great advantage over the other previous method $[9,10,12]$. Here, the cost means the extra bits required to hide the secret data and the neat capacity represents the difference value between the total hiding capacity and the cost. All of them are measured by the number of bits. 


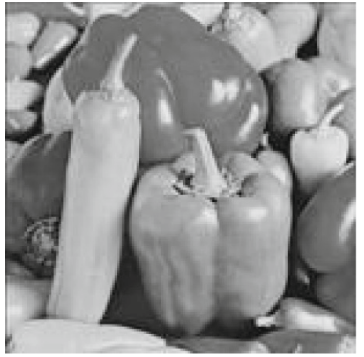

(a) Peppers

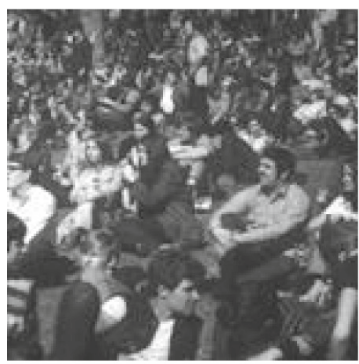

(d) People

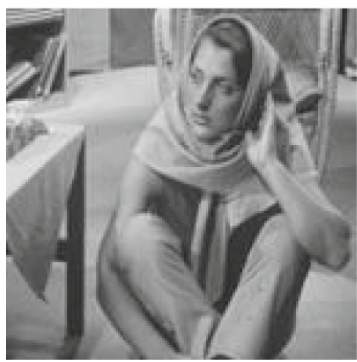

(g) Barbara

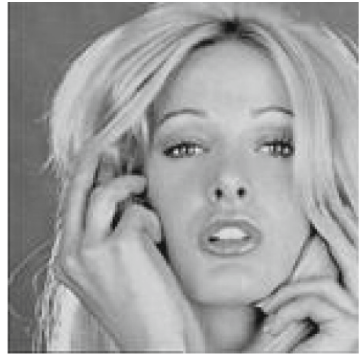

(b) Tiffany

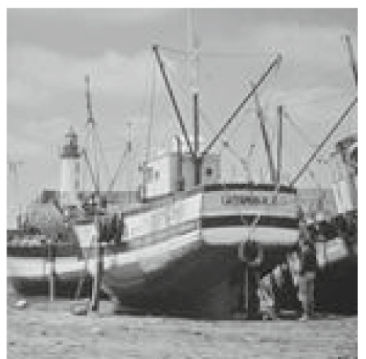

(e) Boat

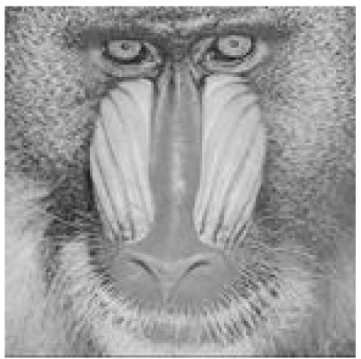

(h) Baboon

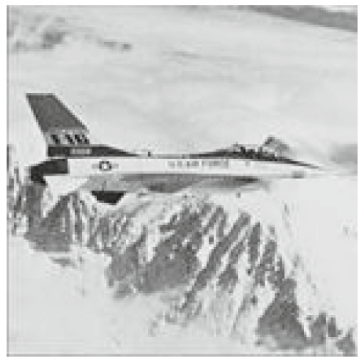

(c) Jet

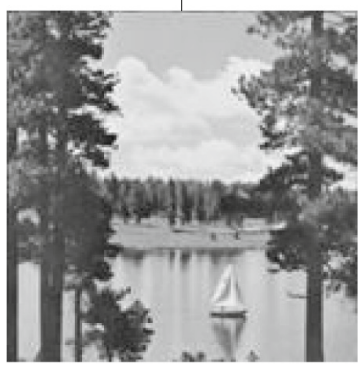

(f) Lake

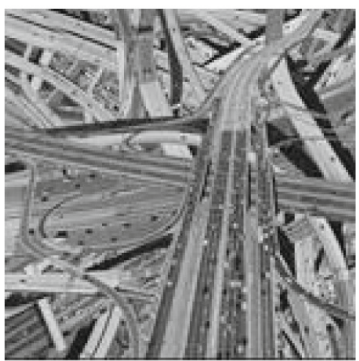

(i) Bridge

Fig. 2. Test images

In order to illustrate the superiority of our proposed method, the comparison with Tu's scheme has been shown in Table 1 in terms of total capacity, cost and the neat capacity under different codebook sizes which are 256, 512, and 1024, respectively. According to Table 1, the total capacity of hidden secret bits is not affected regardless of what kind of image it is. Additionally, the payload of our proposed method, which reaches 16384 bits, is as high as that of Tu's scheme. The cost is gradually reduced as the codebook size increases. For smooth images, the cost of our scheme is a little more than Tu's method with quite small differences. However, our scheme is more suitable for complex images with abundant textures. The advantage on cost will be much more obvious as the complexity of texture increases. For the extremely high texture images 'Baboon' and 'Bridge', it reduces approximately more than one thousand bits than Tu's scheme. The experimental results reveal that the proposed scheme can yield much better performance than Tu's method. 
Table 1. The comparisons of Tu's scheme and the proposed scheme in terms of capacity, cost and neat capacity

\begin{tabular}{|c|c|c|c|c|c|c|c|c|c|c|}
\hline \multirow{3}{*}{\multicolumn{2}{|c|}{$\begin{array}{l}\text { Codebook } \\
\text { size }\end{array}$}} & \multicolumn{9}{|l|}{ Image } \\
\hline & & \multicolumn{3}{|l|}{ Smooth } & \multicolumn{6}{|c|}{ Complex } \\
\hline & & Peppers & Tiffany & Jet & People & Boat & Lake & Barbara & Baboon & Bridge \\
\hline \multirow[t]{8}{*}{256} & Proposed & & & & & & & & & \\
\hline & Capacity & 16384 & 16384 & 16384 & 16384 & 16384 & 16384 & 16384 & 16384 & 16384 \\
\hline & Cost & 2639 & 2723 & 3041 & 3849 & 3862 & 4621 & 4931 & 9657 & 10901 \\
\hline & Neat & 13745 & 14561 & 13343 & 12626 & 12522 & 11763 & 11453 & 6727 & 5483 \\
\hline & Tu's & & & & & & & & & \\
\hline & Capacity & 16384 & 16384 & 16384 & 16384 & 16384 & 16384 & 16384 & 16384 & 16384 \\
\hline & Cost & 2580 & 2702 & 3126 & 4238 & 4584 & 5238 & 5682 & 11024 & 12680 \\
\hline & Neat & 13804 & 13682 & 13258 & 12146 & 11800 & 11146 & 10702 & 5360 & 3704 \\
\hline \multirow[t]{8}{*}{512} & Proposed & & & & & & & & & \\
\hline & Capacity & 16384 & 16384 & 16384 & 16384 & 16384 & 16384 & 16384 & 16384 & 16384 \\
\hline & Cost & 2330 & 2572 & 2947 & 3203 & 3368 & 4239 & 4175 & 9424 & 9835 \\
\hline & Neat & 14054 & 13812 & 13437 & 13181 & 13016 & 12145 & 12209 & 6960 & 6549 \\
\hline & Tu's & & & & & & & & & \\
\hline & Capacity & 16384 & 16384 & 16384 & 16384 & 16384 & 16384 & 16384 & 16384 & 16384 \\
\hline & Cost & 2336 & 2520 & 2998 & 3590 & 3974 & 4778 & 4562 & 11032 & 11836 \\
\hline & Neat & 14048 & 13864 & 13414 & 12794 & 12410 & 11606 & 11822 & 5352 & 4548 \\
\hline \multirow[t]{8}{*}{1024} & Proposed & & & & & & & & & \\
\hline & Capacity & 16384 & 16384 & 16384 & 16384 & 16384 & 16384 & 16384 & 16384 & 16384 \\
\hline & Cost & 2048 & 2341 & 2794 & 2689 & 2914 & 4078 & 4016 & 9269 & 9478 \\
\hline & Neat & 14336 & 14043 & 13590 & 13695 & 13470 & 12306 & 12368 & 7115 & 6906 \\
\hline & Tu's & & & & & & & & & \\
\hline & Capacity & 16384 & 16384 & 16384 & 16384 & 16384 & 16384 & 16384 & 16384 & 16384 \\
\hline & Cost & 1960 & 2246 & 2726 & 2922 & 3474 & 4466 & 4356 & 10874 & 10902 \\
\hline & Neat & 14424 & 14138 & 13658 & 13462 & 12910 & 11918 & 12128 & 5510 & 5482 \\
\hline
\end{tabular}




\section{Conclusions}

In this paper, we propose a novel reversible data hiding scheme using ordered clusterbased VQ code index for grayscale images, which is of low computational complexity. Specifically, the number of secret bits can be embedded into an image is decided by the number of segmented blocks divided by VQ algorithm. Our method outperforms Tu's scheme by means of alleviating extra bit consumption needed to hide secret data. Furthermore, we can reconstruct the VQ compressed images without any distortion after secret data extraction. Performance comparisons based on nine test images demonstrate that the proposed scheme can achieve lower bit cost under a relatively high payload for complex grayscale images under various codebook sizes.

\section{References}

1. Wu, M., Liu, B.: Data hiding in image and video: part I-fundamental issues and solutions. IEEE Trans. Image Process. 12(6), 685-695 (2003)

2. Schyndel, R.G.V., Tirkel, A.Z., Osborne, C.F.: A digital watermark. In: Proceedings of IEEE International Conference on Image Processing (ICIP), vol. 2, pp. 86-90 Austin, TX November 1994

3. Chan, C.K., Cheng, L.M.: Hiding data in images by simple LSB substitution. Pattern Recogn. 37(3), 469-474 (2004)

4. Mielikanen, J.: LSB matching revisited. IEEE Signal Process. Lett. 13(5), 285-297 (2006)

5. Linde, Y., Buzo, A., Gray, R.M.: An algorithm for vector quantizer design. IEEE Trans. Commun. 28(1), 84-95 (1980)

6. Zhu, C., Hua, Y.: Image vector quantization with minimax Lo distortion. IEEE Signal Process. Lett. 6(2), 25-27 (1999)

7. Zhu, C., Po, L.M.: Partial distortion sensitive competitive learning algorithm for optimal codebook design. Electron. Lett. (IEE) 32(19), 1757-1758 (1996)

8. Zhu, C., Po, L.M.: Minimax partial distortion competitive learning for optimal codebook design. IEEE Trans. Image Process. 7(10), 1400-1409 (1998)

9. Jo, M., Kim, H.D.: A digital image watermarking scheme based on vector quantization. IEICE Trans. Inf. Syst. 85(6), 1054-1056 (2002)

10. Chang, C.C., Wu, W.C., Hu, Y.C.: Lossless recovery of a VQ index table with embedded secret data. J. Vis. Commun. Image Represent. 18(3), 207-216 (2007)

11. Tu, T.Y., Wang, C.H.: Reversible data hiding with high payload based on referred frequency for VQ compressed codes index. Sig. Process. 108, 278-287 (2015)

12. Chang, C.C., Tai, W.L., Lin, M.H.: A reversible data hiding scheme with modified side match vector quantization. In: Proceedings of IEEE International Conference on Advanced Information Networking and Applications (AINA), vol. 1, pp. 947-952 Taipei, Taiwan March 2005 